The Astrophysical Journal, 222: 281-296, 1978 May 15

(C) 1978. The American Astronomical Society. All rights reserved. Printed in U.S.A.

\title{
SECULAR INSTABILITY OF ROTATING NEWTONIAN STARS*
}

\author{
JoHn L. Friedman \\ Department of Physics, University of Wisconsin-Milwaukee \\ AND \\ BERNARD F. SCHUTZ \\ Department of Applied Mathematics and Astronomy, University College, Cardiff, Wales \\ Received 1977 July 11; accepted 1977 November 7
}

\begin{abstract}
The effect of gravitational radiation and of viscosity on the stability of rotating self-gravitating fluids is considered. Previous criteria governing secular stability to radiation are shown to fail as a result of the trivial displacements introduced in a previous paper (I). The required modification is obtained by describing perturbations in terms of canonical displacements (displacements orthogonal to the trivials). There nevertheless remain physical perturbations having angular dependence $e^{i m \phi}$ which, for sufficiently large $m$, make the stability functional (the canonical energy $E_{c}$ ) negative, and it follows that all rotating stars are unstable or marginally unstable to gravitational radiation. In the case of stability against viscosity, the corresponding stability criterion is shown to involve the canonical energy in a rotating frame, $E_{c, R}$, a functional invariant under gauge transformations associated with the trivial displacements. By using the functional $E_{c, R}$ to analyze local stability, it is found that a star is locally stable against viscosity if and only if the specific entropy increases outward (in the sense of decreasing pressure). Finally, the behavior of normal modes is discussed and used to elucidate the generic radiation-induced instability; and certain orthogonality properties are derived.
\end{abstract}

Subject headings: gravitation — instabilities - stars: rotation

\section{INTRODUCTION}

This is the second of two papers dealing with perturbations and stability of nearly Newtonian stars. In this paper we apply the Lagrangian perturbation theory developed in Paper I (Friedman and Schutz 1978) to the problem of secular instability of rotating stars arising from radiation reaction and from viscosity.

It had long been thought (Thompson and Tait 1883) that models of rotating stars which are stable in the absence of dissipation can nevertheless be unstable when viscosity is present. The fact was not explicitly demonstrated, however, until 1963, when Roberts and Stewartson showed that in the presence of slight viscosity the Maclaurin sequence is unstable to a bar mode beyond the bifurcation point where the mode has zero frequency in the rotating frame. The phenomenon is due to the fact that viscous forces violate a constraint on the perfect fluid-namely, the conservation of circulation in constant entropy surfaces-and that viscosity can thereby permit transitions to lower energy states that would be inaccessible to a perfect fluid. In 1970, Chandrasekhar found that gravitational radiation gives rise to a similar instability in Maclaurin spheroids, which one could ascribe in an analogous way to the angular momentum nonconservation of the radiation reaction force. Subsequent work found that the radiation-induced instability could be important in models of rapidly rotating white dwarfs and neutron stars (Friedman and Schutz 1975b; Durisen 1975). The growth time for these instabilities is determined by the dissipative mechanisms and is generally long compared to the dynamical time scale.

The 1967 paper of Lynden-Bell and Ostriker proposed a general criterion for deciding whether a stellar model was unstable in the presence of a dissipative force. Perturbations of an equilibrium model were described by means of a Lagrangian displacement, and a configuration was claimed to be stable if and only if a certain operator $C^{i}{ }_{j}$ (also the $C^{i}{ }_{j}$ of Paper I) was positive definite. This condition on $C^{i}{ }_{j}$ is equivalent to demanding that the canonical energy $E_{c}$ of the perturbation be positive definite for all nonzero initial data. Later work by Tassoul, Ostriker, and Bodenheimer (Tassoul and Ostriker 1968; Ostriker and Tassoul 1969; Ostriker and Bodenheimer 1973) used tensor virial techniques to test stability of particular differentially rotating stars, in effect evaluating the LyndenBell-Ostriker functional, $\int \xi^{*}{ }_{i} C^{i}{ }_{j} \xi^{j} d V$, for a specific trial displacement $\xi^{i}$ linear in the Cartesian coordinates.

There are, however, several problems with these related approaches. First, Hunter (1977) has observed that instability in the presence of viscosity will not in general set in at the same point along a sequence of rotating stars as instability to radiation reaction, and so separate criteria are required for the two cases. A second difficulty is associated with the existence of trivial Lagrangian displacements which do not change the physical configuration

* Supported in part by NSF grant MPS 74-17456. 
but whose canonical energy is nonetheless negative. Such displacements violate the Lynden-Bell-Ostriker criterion, although of course they do not imply an instability. We regain a criterion for secular stability to gravitational radiation by requiring that perturbations be described by the canonical displacements-displacements orthogonal to the trivials-introduced in Paper I. The tensor-virial displacement mentioned above is not orthogonal to the trivials, and consequently the tensor-virial criterion will not in general guarantee secular instability. On the other hand, when viscosity is the dissipative mechanism, we will see that the corresponding stability criterion involves a functional that vanishes for the trivial displacements-in this case the trivials are harmless, but the criterion is again inequivalent to the old tensor-virial one.

The plan of the paper is as follows. In $\S$ II we consider secular instability to radiation reaction and find that when one admits only canonical displacements, positivity of the canonical energy $E_{c}$ provides the desired stability criterion. Using this criterion, we find the remarkable result that all rotating, self-gravitating perfect fluids are unstable or marginally unstable to gravitational radiation. It had previously been thought that instability along a sequence of rotating stars would first set in via a bar mode, a mode having angular dependence of the form $e^{2 i \phi}$, and that at the point of marginal stability the ratio of kinetic to potential energy $T /|W|$ would be about 0.14 (Ostriker and Tassoul 1969). We find, however, that models with arbitrarily slow rotation have unstable multiarm oscillations with angular dependence $e^{i m \phi}$ for sufficiently large $m$. On the other hand, because the time scale for the multiarm instability is likely to be too long for these modes to be important, the $m=2$ mode probably remains the physically interesting phenomenon. In the final part of $\S$ II, we review the tensor-virial method for locating the $m=2$ instability point and show that the method will not in general imply instability.

Section III deals with secular instability to viscosity. For differentially rotating stars the notion of a viscosityinduced instability may be empty in that the growth time of such an instability would be comparable to the time needed to alter substantially the equilibrium configuration by viscous readjustment of its angular velocity. For uniformly rotating stars, however, the question of viscous instability is meaningful. In this case, the canonical energy defined in the rotating frame, $E_{c, R}$, is a decreasing function of time and can be used to phrase a stability criterion analogous to that introduced previously for the radiation-induced instability. Moreover, the functional $E_{c, R}$ is invariant under the gauge transformations associated with trivial displacements: as we saw in Paper I, $E_{c, R}$ depends only on the physical perturbation, and one need not use canonical displacements (in fact, the criterion can be stated in an entirely Eulerian framework). Using the criterion, we find that a necessary and sufficient condition for local secular stability is that the specific entropy $s$ decrease outward (in the direction of decreasing pressure). This naturally generalizes the Schwarzschild criterion for local (dynamical) instability of nonrotating stars.

In $\S$ II and III, no assumptions are made concerning the existence or completeness of normal modes, perturbations having harmonic time dependence. It is not unlikely, however, that it will be commonly possible to decompose an arbitrary perturbation (of a nondissipative fluid) into a sum of modes corresponding to a continuous real spectrum of frequencies together with a discrete set of complex frequencies associated with dynamically unstable modes. For this reason, and because a normal mode analysis provides a clearer physical description of radiationinduced instability, we consider in $\S$ IV the application of our results to normal modes of oscillation. In particular, we show that instability to gravitational radiation sets in along a sequence of rotating stars when a counterrotating mode changes the sign of its pattern speed and becomes corotating; this behavior is so common in rotating systems coupled to radiation that it leads us to identify a new class of instabilities, the rotational dragging instabilities. In the last part of $\S I V$, we show that normal modes with no corotating points are automatically orthogonal to the trivial displacements and that modes having definite frequencies are orthogonal to each other (with respect to the inner product $W$ of Paper I).

Finally, in $\S V$, we list a number of problems suggested by our work.

\section{INSTABILITY TO GRAVITATIONAL RADIATION}

The question of secular stability of Newtonian stars is really the question of whether nearly Newtonian stars are unstable in the exact framework of general relativity. In relativistic stability theory, as in the Newtonian theory, one describes perturbations of a fluid equilibrium by means of a Lagrangian displacement. In this way one obtains an action for the perturbation equations and (via Noether's theorem) a conserved current $J^{a}$ associated with the time independence of the equilibrium configuration. The canonical energy may then be defined by

$$
E_{c}=\int_{V} J^{a} d S_{a}
$$

where the integral is evaluated on a spacelike hypersurface-say $t=t_{0}$. Now the conservation of $J^{a}$ implies that the difference in $E_{c}$ from one spacelike (but asymptotically null) hypersurface $t=t_{0}$ to another $t=t_{1}$ in its future is just the energy radiated to infinity between $t_{0}$ and $t_{1}$ by gravitational waves. Because this radiated energy is positive (or zero), $E_{c}$ is a decreasing function of time. For nearly Newtonian stars, the functional $E_{c}$ is the canonical 
energy given in Paper I (I.6), and is a quadratic function of the Lagrangian displacement $\xi$ and its first time derivative $\dot{\xi}$ at a given time. ${ }^{1}$

In linearized perturbation theory, unstable nonaxisymmetric perturbations of an axisymmetric background will grow without bound, radiating infinite energy. Stable perturbations, on the other hand, can radiate only finite energy; and one expects that they will die away in time - that the star will radiate until it settles back down to an axisymmetric configuration. Now, the functional $E_{c}$ depends only on the initial data $(\xi, \dot{\xi})$ for the perturbation at a given time. Thus if $E_{c}$ were positive for all initial data at a time $t$, then it would have to be positive for all data at any later time as well (because of the time independence of the equilibrium, the functional form of $E_{c}$ is independent of time); and in that case no perturbation could radiate more energy than the initial value of $E_{c}$ at $t$. If, on the other hand, $E_{c}$ could be made negative for some initial data, then-since its value can only decreaseit will never return to zero, and it would appear that the corresponding perturbation could not die away in time. In other words, the functional $E_{c}$ would seem to govern stability to nonaxisymmetric perturbations in the following sense:

i) If $E_{c}(\xi) \geq 0$ for all initial data, the configuration is stable in the sense that no perturbation radiates infinite energy.

ii) If $E_{c}(\xi)<0$ for some initial data, the configuration is unstable or marginally unstable: there exist nonaxisymmetric perturbations which do not die away in time.

As stated, however, the stability criteria (i) and (ii) overlook the existence of trivial Lagrangian displacements. That is, as we showed in Paper I, there is a class of displacements that leaves all the physical variables unchanged but for which the canonical energy $E_{c}$ is nevertheless nonzero. Thus (i) is true but vacuous: one can always find initial data for a trivial displacement that makes $E_{c}<0$. Similarly, statement (ii) is false: the fact that $E_{c}<0$ for some displacement will not in general signal an instability because a physical perturbation that dies away in time can be described by a displacement that remains finite, evolving to a trivial for which $E_{c}<0$.

In order to regain a stability criterion, it is necessary to restrict the class of allowed displacements so that $E_{c} \neq 0$ will imply that the physical perturbation is itself nonvanishing. Fortunately, as we saw in Paper I (§ IV) there is a class of canonical displacements, naturally distinguished by the structure of the theory and such that (a) the class is dynamically invariant and $(b)$ if $\xi$ and $\hat{\xi}$ are two canonical displacements describing the same physical situation, then $E_{c}(\xi)=E_{c}(\xi)$. For nonisentropic stars, in fact, the assignment of canonical Lagrangian displacements to physical perturbations is unique; for isentropic stars, a slight freedom of choice remains but the freedom does not affect $E_{c}$. Conversely, for isentropic stars there exists a canonical displacement corresponding to each physical displacement; but it appears that for some nonisentropic stars, ${ }^{2}$ restricting consideration to canonical displacements will be a real restriction on the allowed physical perturbations.

Explicitly, a displacement $\xi^{i}$ is canonical if it is orthogonal to all trivial displacements with respect to the inner product $W$, the symplectic structure introduced in Paper I (I.39). That is,

$$
W(\xi, \eta) \equiv \int\left[\rho \xi^{i}\left(\dot{\eta}_{i}+v \cdot \nabla \eta_{i}\right)-\rho \eta^{i}\left(\dot{\xi}_{i}+v \cdot \nabla \xi_{i}\right)\right] d V=0
$$

for all trivial displacements $\eta^{i}$. For nonisentropic stars the orthogonality condition is equivalent to requiring that the displacement $\xi$ preserve the vorticity of each fluid element in surfaces of constant entropy-formally, that

$$
\epsilon^{i j k} \nabla_{i} s \nabla_{j} \Delta_{\xi} v_{k}=0,
$$

where the Lagrangian change in the velocity $v_{i}$ is

$$
\Delta_{\xi} v_{i}=\dot{\xi}_{i}+v^{j}\left(\nabla_{j} \xi_{i}+\nabla_{i} \xi_{j}\right) .
$$

For isentropic stars, the class of trivials is larger and the condition is more restrictive, namely, that all components of the vorticity be preserved for each fluid element, or

$$
\nabla_{i} \Delta_{\xi} v_{j}-\nabla_{j} \Delta_{\xi} v_{i}=0
$$

By canonical initial data, we will mean a pair of vector fields $\left(\xi^{i}, \dot{\xi}^{i}\right)$ which satisfy equation (2), or, in the isentropic case, (4). Because $W(\xi, \eta)$ is time independent when $\xi$ and $\eta$ are solutions to the dynamical equation (I.15), the time evolution of canonical initial data is a canonical displacement. In fact even in the presence of gravitational radiation reaction, $W(\xi, \eta)$ is conserved when either $\xi$ or $\eta$ is trivial. Thus, in particular, the condition that $\xi$ be canonical is dynamically preserved. This fact is related to the existence of an exactly conserved quantity for relativistic fluids analogous to the Newtonian circulation in surfaces of constant entropy.

\footnotetext{
${ }^{1}$ Technically, the relativistic $E_{c}$ has as its Newtonian limit two terms. The first can be identified with the energy of gravitational waves on flat space and involves initial data independent of the fluid perturbation; this is positive definite and so is uninteresting from the point of view of stability theory. The second term of the relativistic functional is the Newtonian canonical energy of Paper I (see, e.g., Friedman and Schutz 1975a, Appendix I).

${ }^{2}$ Explicitly, when $\nabla \alpha \times \nabla s=0$, where $s$ is the specific entropy and $\alpha=\nabla s \cdot \nabla \times v=s, w\left(w^{2} \Omega\right)_{, z}-s_{, z}\left(w^{2} \Omega\right), w$. See (I.80).
} 
A sufficient condition for instability may now be phrased in the following manner:

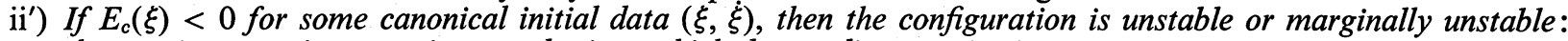
there exist nonaxisymmetric perturbations which do not die away in time.

Similarly, for isentropic stars, and for nonisentropic stars in which $\nabla \alpha \times \nabla s \neq 0$, a sufficient condition for stability is:

$\left.i^{\prime}\right)$ If $E_{c}(\xi) \geq 0$ for all canonical initial data, the configuration is stable in the sense that no perturbation radiates infinite energy, and the magnitude of $E_{c}$ is bounded.

The apparent lack of a sufficient condition for stability, in the case where $\nabla \alpha \times \nabla s=0$ somewhere in the equilibrium star, is mitigated by the fact that (as we show below) all rotating stars are unstable in any event.

\section{a) Generic Instability of Rotating Nonisentropic Fluids}

We will first consider nonisentropic configurations, perfect fluids having equations of state of the form $p=$ $p(\rho, s)$. We will pick canonical initial data $(\xi, \dot{\xi})$ having angular dependence $e^{i m \phi}$ (where $\phi$ is the angle about the symmetric axis) and will show that $E_{c}(\xi)<0$ when the integer $m$ is sufficiently large. When $\xi$ is complex, the canonical energy has the form given by equation (I.45),

$$
\begin{gathered}
E_{c}(\xi)=\frac{1}{2} \int\left[\rho|\dot{\xi}|^{2}-\rho|v \cdot \nabla \xi|^{2}+\gamma p|\nabla \cdot \xi|^{2}+\xi^{*} \cdot \nabla p \nabla \cdot \xi+\xi \cdot \nabla p \nabla \cdot \xi^{*}\right. \\
\left.+\xi^{i} \xi^{j *}\left(\nabla_{i} \nabla_{j} p+\rho \nabla_{i} \nabla_{j} \Phi\right)-\frac{1}{4 \pi G}|\nabla \delta \Phi|^{2}\right] d V,
\end{gathered}
$$

where the Eulerian change in the potential, $\delta \Phi$, is defined by the equation

$$
\nabla^{2} \delta \Phi=-4 \pi G \nabla \cdot(\rho \xi) .
$$

Suppose that as initial data we choose a pair $\left(\xi^{i}, \dot{\xi}^{i}\right)$ having the form given in cylindrical coordinates by

$$
\xi^{i}=\zeta^{i}(\varpi, z) e^{i m \phi}, \quad \dot{\xi}^{i}=\dot{\zeta}^{i}(\varpi, z) e^{i m \phi},
$$

where $\zeta^{\phi}=0$. The vectors $\zeta^{i}$ and $\dot{\zeta}^{i}$ may depend on the integer $m$, but we will require that the magnitudes of $\zeta^{i}$ and its first derivatives and of $\dot{\zeta}^{i}$ are bounded everywhere by some constant $C$ independent of $m$. Then the divergence of $\xi^{i}$ has the form

$$
\nabla \cdot \xi=\nabla \cdot \zeta e^{i m \phi},
$$

and so it too will be bounded by a constant independent of $m$. Now if the equilibrium fluid variables $p, \rho$, and $\Phi$ are smooth (if they have bounded second derivatives), the terms

$$
\rho|\dot{\xi}|^{2}, \quad \gamma p|\nabla \cdot \xi|^{2}, \quad \xi^{*} \cdot \nabla p \nabla \cdot \xi+\xi \cdot \nabla p \nabla \cdot \xi^{*}, \quad \text { and } \quad \xi^{i} \xi^{j}\left(\nabla_{i} \nabla_{j} p+\rho \nabla_{i} \nabla_{j} \Phi\right)
$$

appearing in the integrand of equation (5) for $E_{c}$ will all be similarly bounded. The only remaining terms,

$$
-\rho|v \cdot \nabla \xi|^{2} \quad \text { and } \quad-\frac{1}{4 \pi}|\nabla \delta \Phi|^{2},
$$

are negative definite, ${ }^{3}$ and thus

$$
E_{c} \leq-\int \rho|v \cdot \nabla \xi|^{2}+K
$$

where again $K$ is independent of the angular eigenvalue $m$. Finally

whence

$$
|v \cdot \nabla \xi|^{2}=m^{2} \Omega^{2}|\zeta|^{2}+\Omega^{2}\left|\zeta^{w}\right|^{2} \geq m^{2} \Omega^{2}|\zeta|^{2}
$$

$$
E_{c} \leq-m^{2} \int \rho \Omega^{2}|\zeta|^{2} d V+K
$$

For $m$ sufficiently large, $E_{c}$ will be negative (as long as $\rho \Omega^{2}|\zeta|^{2}$ is not everywhere zero).

A particular choice of canonical initial data of the form (7) is given by

$$
\zeta_{i}=f \partial_{i} s, \quad \dot{\zeta}_{i}=\frac{2}{i m} \partial_{i}\left(\varpi \Omega f \partial_{w} s\right),
$$

${ }^{3}$ The quantity $|\nabla \delta \Phi|^{2}$ is also bounded by a constant independent of $m$, but the fact is not needed here. 
where $f$ is a smooth function of $w$ and $z$ vanishing in some neighborhood of the axis (where $\varpi=0$ ). To see that the data are canonical - that is, satisfy the orthogonality condition (2)-we rewrite the condition in terms of $\zeta^{i}$ as follows. The Lagrangian change in the velocity (3) takes the form

$$
\Delta_{\xi} v_{i}=\partial_{t}\left(\zeta_{i} e^{i m \phi}\right)+v^{j} \nabla_{j}\left(\zeta_{i} e^{i m \phi}\right)+v^{j} \nabla_{i}\left(\zeta_{j} e^{i m \phi}\right)=\left[\partial_{t} \zeta_{i}+i m \Omega \zeta_{i}+2 \varpi \Omega \zeta_{\varpi} \delta_{i}^{\phi}\right] e^{i m \phi} .
$$

Then

Now

$$
\epsilon^{i j k} \nabla_{i} s \nabla_{j} \Delta_{\xi} v_{k}=\epsilon^{i j k} \partial_{i} s \partial_{j} \Delta_{\xi} v_{k}=\epsilon^{i j k} \partial_{i} s \partial_{j}\left[\left(\partial_{t} \zeta_{k}+i m \Omega \zeta_{k}+2 \varpi \Omega \zeta_{\varpi} \delta_{k}^{\phi}\right) e^{i m \phi}\right] .
$$

(because $\left.\zeta_{k}=f \nabla_{k} s\right)$, and

$$
\begin{aligned}
& \epsilon^{i j k} \partial_{i} s \partial_{j}\left(\partial_{i} \zeta_{k} e^{i m \phi}\right)=\frac{i m}{\varpi}\left(\dot{\zeta}_{\varpi} \partial_{z} s-\dot{\zeta}_{z} \partial_{\varpi} s\right) e^{i m \phi} \\
& \epsilon^{i j k} \partial_{i} s \partial_{j}\left(\Omega \zeta_{k} e^{i m \phi}\right)=0
\end{aligned}
$$

$$
\epsilon^{i j k} \partial_{i} s \partial_{j}\left(2 \varpi \Omega \zeta_{\varpi} \delta_{k}^{\phi}\right)=\frac{2}{\varpi}\left[\partial_{\varpi} s \partial_{z}\left(\varpi \Omega \zeta_{\varpi}\right)-\partial_{z} s \partial_{w}\left(\varpi \Omega \zeta_{\varpi}\right)\right] ;
$$

and the orthogonality condition becomes

$$
\begin{aligned}
0 & =e^{-i m \phi} \epsilon^{i j k} \partial_{i} s \partial_{j} \Delta_{\xi} v_{k} \\
& =\frac{1}{\varpi}\left[i m \dot{\zeta}_{w}-2 \partial_{\varpi}\left(\varpi \Omega \zeta_{\varpi}\right)\right] \partial_{z} s-\frac{1}{\varpi}\left[i m \dot{\zeta}_{z}-2 \partial_{z}\left(\varpi \Omega \zeta_{\varpi}\right)\right] \partial_{\varpi} s .
\end{aligned}
$$

For the initial data given in equation (11), the coefficients of $\partial_{z} s$ and $\partial_{\varpi} s$ vanish separately, and condition (2) is satisfied. We conclude that the pair

$$
\xi_{i}=f \partial_{i} s e^{i m \phi}, \quad \dot{\xi}_{i}=\frac{2}{i m \omega} \partial_{i}\left(\varpi \Omega f \partial_{\varpi} s\right) e^{i m \phi}
$$

are canonical initial data for which

$$
E_{c}(\xi) \leq-m^{2} \int \rho \Omega^{2}|\zeta|^{2}+K
$$

and since $\zeta^{i}$ is itself independent of $m, E_{c}(\xi)<0$ for all $m$ greater than some $m_{0}$.

Some editorial comment is in order concerning this result. First, since all that has been proved is that a set of nonaxisymmetric perturbations will not die away in time, one might ask whether a real instability is indicated. We have little doubt that rotating fluids are in fact strictly unstable to radiation reaction, and the argument is this. Each initial data set with angular dependence $e^{i m \phi}$ for each $E_{c}<0$ must either (1) approach a stationary nonaxisymmetric state, implying the existence of a zero frequency mode for that value of $m$, (2) approach a timedependent but nonradiative state (whose time derivatives remain finitely large forever), or (3) radiate an infinite amount of energy. Alternative (1) is the way instability commonly sets in in a particular mode (Friedman and Schutz 1975a); but it is rare that a configuration has any zero-frequency mode and highly unlikely that a star would have zero-frequency modes for all values of $m$ greater than some $m_{0}$. The second alternative would be ruled out if one could show that any pulsation involved a nonzero density perturbation; for in that case the $m$ th mass multipole moment would change in time and the system would radiate. This is automatically true for isentropic fluids because $\delta \rho$ vanishes only for trivial displacements. In more general fluids, the dynamical equations probably rule out time-dependent perturbations for which $\delta \rho=0$; but if there were pulsations for which only $\delta p$ and $\delta s$ were nonzero, one would expect that they too would radiate, losing energy at a rate slower than that for a density perturbation by a factor on the order of $p / \rho c^{2}$.

One is thereby led to the third alternative, representing a strict instability in the linearized theory. In the exact theory, one expects a perturbed rotating fluid gradually to radiate away its angular momentum, and to settle down to a spherical, nonrotating configuration.

From a physical point of view, a more serious objection is that the time scale for energy loss due to high multipole moment oscillations is inordinately slow. That is,

$$
\frac{d E}{d t} \sim\left(\frac{d^{m+1}}{d t^{m+1}} Q_{m}\right)^{2},
$$

where $Q_{m}$ is the $m$ th mass multiple moment, and it follows that the $e$-folding time exceeds the dynamical time by a factor $(c / v)^{2 m+1}$, where $v$ is a characteristic velocity of the perturbation. ${ }^{4}$ Thus for slowly rotating stars the

${ }^{4}$ For the ergoregion instability of a scalar field, which has many formal similarities to this one, Comins and Schutz (1978) find that for large $m$ the $e$-folding time is $\tau=$ const. $\times \exp (\alpha m)$. 
instability is unimportant. For nearly-Newtonian configurations rotating rapidly enough that high $m$ modes could play a role, the $m=2$ mode might already be unstable, in which case it would dominate. The physical limitation on rotation would then continue to be set by the Dedekind-like bar mode-the onset of instability along a sequence of rotating stars would effectively not be far from the first point at which an $m=2$ mode has zero frequency.

Finally, recent work by Lindblom and Detweiler (1977) on the evolution of Maclaurin spheroids in the presence of both radiation reaction and viscosity indicates that when the viscous and radiation-reaction time scales are comparable, the two dissipative mechanisms can damp the instability that each drives separately: This suggests that viscosity may stabilize modes with growth times long compared to the time for viscous readjustment of the velocity profile, and thereby eliminate the high $m$ instability in slowly rotating stars. (Our result is mathematically similar to an analysis of accretion disks due to Stewart 1975, but his work has several flaws: see $\S$ III $c$ below.)

\section{b) Generic Instability of Isentropic Fluids}

The demonstration of a generic instability of isentropic fluids differs somewhat from that given above for stellar models having a nonzero entropy gradient. The structure of the instability is also somewhat more restricted, arising only in regions where the fluid velocity exceeds the speed of sound in the star. (Such regions always exist because $v_{s}$ vanishes at the surface but $\Omega$, which is constant on cylinders, does not.)

Canonical displacements $\xi^{i}$ of isentropic stars satisfy

or

$$
\begin{gathered}
\nabla_{i} \Delta_{\xi} v_{j}-\nabla_{j} \Delta_{\xi} v_{i}=0, \\
\Delta_{\xi} v_{i}=\dot{\xi}_{i}+v^{j}\left(\nabla_{j} \xi_{i}+\nabla_{i} \xi_{j}\right)=-\nabla_{i} f
\end{gathered}
$$

for some scalar $f$. Specifying general canonical data $\left(\xi^{i}, \dot{\xi}^{i}\right)$ thus amounts to picking a vector $\xi^{i}$ and a scalar $f$; $\dot{\xi}^{i}$ is then determined by equation (18b). The canonical energy $E_{c}$ may in this way be written explicitly in terms of the unconstrained data, the quantities $\xi^{i}$ and $f$.

It will also be convenient to assume an angular dependence $e^{i m \phi}$ and to write the $\phi$ component of $\xi^{i}$ separately. We will write

and

$$
f=\lambda e^{i m \phi}
$$

$$
\xi^{i}=\left(\mu \delta_{\phi}^{i}+\nu^{i}\right) e^{i m \phi},
$$

where, in cylindrical coordinates, the quantities $\lambda, \mu$, and $\nu^{i}$ depend only on $\varpi$ and $z$ and where $\nu^{\phi}=0$. In order to obtain the functional $E_{c}$ in terms of $\lambda, \mu$, and $\nu^{i}$, we will first find expressions for $|\dot{\xi}|^{2},|v \cdot \nabla \xi|^{2}$, and $\nabla \cdot \xi$. These terms involve the gradient of the displacement vector, which from equation (20) has the form

$$
\nabla_{j} \xi^{i}=\left[i m \delta_{j}^{\phi}\left(\mu \delta_{\phi}^{i}+\nu^{i}\right)+\nabla_{j} \mu \delta_{\phi}^{i}+\mu\left(\frac{1}{\varpi} \delta_{j}{ }^{w} \delta_{\phi}^{i}-\varpi \delta_{j}^{\phi} \delta_{\varpi}^{i}\right)+\nabla_{j} \nu^{i}\right] e^{i m \phi} .
$$

Then from equation (18), we find

and

$$
\dot{\xi}_{i}=-\left[i m\left(\lambda \delta_{i}{ }^{\phi}+2 \Omega \mu \delta_{i}{ }^{\phi}+\Omega \nu_{i}\right)+\nabla_{i} \lambda+\varpi^{2} \Omega \nabla_{i} \mu+\frac{2}{\varpi} \Omega \nu^{w} \delta_{i}{ }^{\phi}\right] e^{i m \phi},
$$

$$
\begin{aligned}
|\dot{\xi}|^{2}= & m^{2}\left[\frac{1}{\varpi^{2}} \lambda \bar{\lambda}+2 \Omega(\lambda \bar{\mu}+\bar{\lambda} \mu)+4 \varpi^{2} \Omega^{2} \mu \bar{\mu}+\Omega^{2} \nu_{i} \bar{\nu}^{i}\right]+i m \Omega\left[\left(\frac{2}{\varpi} \lambda+4 \varpi \Omega \mu\right) \bar{\nu}^{\varpi}-\left(\frac{2}{\varpi} \bar{\lambda}+4 \varpi \Omega \bar{\mu}\right) \nu^{\varpi}\right. \\
& \left.-\left(\nabla_{i} \lambda+\varpi^{2} \Omega \nabla_{i} \mu\right) \bar{\nu}^{i}+\left(\nabla_{i} \bar{\lambda}+\varpi^{2} \Omega \nabla_{i} \bar{\mu}\right) \nu^{i}\right]+\left|\nabla \lambda+\varpi^{2} \Omega \nabla \mu\right|^{2}+4 \Omega^{2}\left|\nu^{\varpi}\right|^{2}
\end{aligned}
$$

Equation (21) gives

and we have

$$
v^{j} \nabla_{j} \xi^{i}=\left[i m\left(\mu \delta_{\phi}^{i}+\nu^{i}\right)-\varpi \mu \delta_{w}^{i}+\frac{1}{\varpi} v^{\varpi} \delta_{\phi}^{i}\right] \Omega e^{i m \phi}
$$

$$
|v \cdot \nabla \xi|^{2}=\Omega^{2}\left[m^{2}\left(\varpi^{2} \mu \bar{\mu}+\nu_{i} \bar{\nu}^{i}\right)+2 i m \varpi\left(\mu \bar{\nu}^{\varpi}-\bar{\mu} \nu^{\varpi}\right)+\varpi^{2} \mu \bar{\mu}+\nu^{\varpi} \bar{\nu}^{\varpi}\right] .
$$

The divergence of the displacement vector is

$$
\nabla \cdot \xi=(i m \mu+\nabla \cdot \nu) e^{i m \phi},
$$

and the terms in $E_{c}$ involving $\nabla \cdot \xi$ are

$$
\gamma p|\nabla \cdot \xi|^{2}=\gamma p\left[m^{2} \mu \bar{\mu}+\operatorname{im}(\mu \nabla \cdot \bar{\nu}-\bar{\mu} \nabla \cdot \nu)+\nabla \cdot \nu \nabla \cdot \bar{\nu}\right]
$$


and

$$
\xi \cdot \nabla p \nabla \cdot \bar{\xi}+\bar{\xi} \cdot \nabla p \nabla \cdot \xi=i m(\mu \bar{\nu} \cdot \nabla p-\bar{\mu} \nu \cdot \nabla p)+\nu \cdot \nabla p \nabla \cdot \bar{\nu}+\bar{\nu} \cdot \nabla p \nabla \cdot \nu \text {. }
$$

Finally, assembling the various expressions (22), (24), (25), and (26) according to equation (5), we find

$$
\begin{aligned}
E_{c}= & \frac{1}{2} \int\left[m^{2} \rho\left[\frac{1}{\varpi^{2}} \lambda \bar{\lambda}+2 \Omega(\lambda \bar{\mu}+\bar{\lambda} \mu)+3 \varpi^{2} \Omega^{2} \mu \bar{\mu}+\frac{\gamma p}{\rho} \mu \bar{\mu}\right]\right. \\
& +i m\left\{\rho \varpi^{2} \Omega\left[\left(\nabla_{i} \frac{\bar{\lambda}}{\varpi^{2}}+\varpi^{2} \Omega \nabla_{i} \frac{\bar{\mu}}{\varpi^{2}}\right) \nu^{i}-\left(\nabla_{i} \frac{\lambda}{\varpi^{2}}+\varpi^{2} \Omega \nabla_{i} \frac{\mu}{\varpi^{2}}\right) \bar{\nu}^{i}\right]+\gamma p(\mu \nabla \cdot \bar{\nu}-\bar{\mu} \nabla \cdot \nu)+\mu \bar{\nu} \cdot \nabla p-\bar{\mu} \nu \cdot \nabla p\right\} \\
& +\rho\left|\nabla \lambda+\varpi^{2} \Omega \nabla \mu\right|^{2}+\rho \Omega^{2}\left(3 \nu^{\varpi} \bar{\nu}^{\varpi}-\varpi^{2} \mu \bar{\mu}\right)+\gamma p \nabla \cdot \nu \nabla \cdot \bar{\nu} \\
& \left.\left.+\nu \cdot \nabla p \nabla \cdot \bar{\nu}+\bar{\nu} \cdot \nabla p \nabla \cdot \nu+\nu^{i} \bar{\nu}^{j}\left(\nabla_{i} \nabla_{j} p+\rho \nabla_{i} \nabla_{j} \Phi\right)-\frac{1}{4 \pi G}|\nabla \delta \Phi|^{2}\right]\right] d V
\end{aligned}
$$

The coefficient of $m^{2}$ appearing in this expression is minimized for fixed $\mu$ when

$$
\lambda=-2 \varpi^{2} \Omega \mu \text {. }
$$

The coefficient then has the value

$$
\rho\left(-\varpi^{2} \Omega^{2}+\frac{\gamma p}{\rho}\right) \mu \bar{\mu}=\rho\left(v_{s}^{2}-v^{2}\right) \mu \bar{\mu},
$$

where

$$
v_{s}=\left(\frac{d p}{d \rho}\right)^{1 / 2}=\left(\frac{\gamma p}{\rho}\right)^{1 / 2}
$$

is the speed of sound in the fluid. For a given choice of $\mu$ and $\nu$, and with $\lambda$ given by (28), equation (27) implies that

$$
E_{c} \leq m^{2} \frac{1}{2} \int \rho\left(v_{s}^{2}-v^{2}\right) \mu \bar{\mu}+m k_{1}+k_{2},
$$

where $k_{1}$ and $k_{2}$ are constants independent of $m$. Thus, as long as the fluid velocity exceeds the speed of sound somewhere in the star, the function $\mu$ can be taken to be nonzero only where $v>v_{s}$; whence, for sufficiently large $m$, the functional $E_{c}$ will be negative.

Because the adiabatic index $\gamma$ is always greater than one, $v_{s} \rightarrow 0$ when $p \rightarrow 0$ at the surface of the fluid. It follows that there is a region near the boundary of any rotating isentropic star where $v>v_{s}$, and the fluid will therefore be unstable to gravitational radiation. In $\S$ IV we will indicate how the instability in slowly rotating isentropic and nonisentropic stars arises in the $p$ - and $g$-modes of nonrotating models.

\section{c) Problems with the Tensor-Virial Method}

An efficient technique for testing secular stability of differentially rotating stars was developed by Tassoul and Ostriker (1968) and applied by them (Ostriker and Tassoul 1969) to numerical models constructed by Ostriker and Bodenheimer (1968). They made the remarkable discovery that secular instability appeared to set in whenever the ratio $t=T /|W|$ of the kinetic to potential energy of the model exceeded roughly 0.14 , regardless of the rotation law or the (polytropic) equation of state. Similar results have since been found by other investigators (e.g., Durisen 1975; Ostriker and Peebles 1973). Ostriker and Tassoul claimed that their method exactly located the point at which secular instability sets in along a sequence of rotating stellar models. It was subsequently realized, however (Friedman and Schutz 1975b; Hunter 1977), that the method can be exact only when the rotation is uniform, and probably only when the density is uniform as well. One can show without much difficulty that the method locates a model for which the canonical energy of a particular time-independent displacement $\xi_{T}{ }^{i}$ vanishes. In the absence of trivial displacements one could still regard $E_{c}\left(\xi_{T}\right) \leq 0$ as a sufficient condition for instability, but unfortunately the vector $\xi_{T}^{i}$ is not, in general, orthogonal to the trivials. The tensor-virial criterion will therefore not generally guarantee instability, and a modified technique should be developed. Some work in this direction has recently been done by Bardeen (Bardeen et al. 1977), for his thin disk models. By using a canonical displacement having the same Eulerian change in density as the tensor-virial displacement $\xi_{T}$, he finds a value of $t$ about $15 \%$ higher than that provided by the tensor-virial method. This result is in agreement with the location of the first zerofrequency $m=2$ mode.

In the remainder of this section we will briefly review the tensor-virial method and show that it fails to give a sufficient condition for instability - that the tensor-virial displacement is not canonical unless the equilibrium model is uniformly rotating. 
In the tensor-virial method one assumes that a perturbation can be represented as a superposition of normal modes of the form

$$
\xi^{i}=\zeta^{i} e^{i m \phi} e^{i \sigma t}
$$

with complex frequency $\sigma$. The dynamical equation (I.15) then has the form

$$
-\sigma^{2} A^{i}{ }_{j} \xi^{j}+i \sigma B^{i}{ }_{j} \xi^{j}+C^{i}{ }_{j} \xi^{j}=0 \text {. }
$$

Multiplying equation (31) by the Cartesian coordinate $x^{k}$ and integrating over space gives the tensor-virial equations

$$
-\sigma^{2} \int x^{k} A^{i}{ }_{j} \xi^{j} d V+i \sigma \int x^{k} B^{i}{ }_{j} \xi^{j} d V+\int x^{k} C^{i}{ }_{j} \xi^{j} d V=0 .
$$

Tassoul and Ostriker now choose a displacement vector $\xi_{T}{ }^{i}$, whose Cartesian components are linear in the Cartesian coordinates

$$
\xi_{T}^{j}=L^{j}{ }_{i} x^{l},
$$

where $L_{: l}^{j}$ is a constant matrix; and they identify the $m=2$ secular instability point with the first configuration along a sequence of rotating stars for which

$$
0=\int x^{k} C^{i}{ }_{j} \xi_{T}{ }^{j} d V=\left(\int x^{k} C^{i}{ }_{j} x^{l} d V\right) L^{j}{ }_{: l},
$$

where $L_{; l}^{j}$ has the symmetry of an $m=2$ perturbation (the explicit form is given below). Now multiplying equation (33) by $\dot{L}_{i: k}^{*}$ gives

$$
0=\int\left(L_{i ; k} x^{k}\right)^{*} C^{i}{ }_{j}\left(L^{j}{ }_{i} x^{l}\right) d V=\left\langle\xi_{T}, C \xi_{T}\right\rangle
$$

Thus when equation (33) is satisfied, there are initial data, namely $\left(\dot{\xi}_{T}^{i}, \xi_{T}^{i}\right)=\left(0, L_{: j}^{i} x^{j}\right)$ for which

$$
E_{c}\left(\xi_{T}\right) \equiv \frac{1}{2}\left\langle\dot{\xi}_{T}, A \dot{\xi}_{T}\right\rangle+\frac{1}{2}\left\langle\xi_{T}, C \xi_{T}\right\rangle=0,
$$

where we have used equation (I.49) for the canonical energy. The tensor-virial method therefore locates a point along a sequence of rotating stars at which $E_{c}\left(\xi_{T}\right)=0$.

The general form of an $m=2$ displacement vector linear in the Cartesian coordinates is given explicitly by

or

$$
\xi_{T}^{x}=\alpha(x+i y), \quad \xi_{T}^{y}=i \alpha(x+i y), \quad \xi_{T}^{z}=0,
$$

$$
\xi_{T}{ }^{w}=\alpha w e^{2 i \phi}, \quad \xi_{T}^{\phi}=i \alpha e^{2 i \phi}, \quad \xi_{T}^{z}=0 .
$$

The condition that $\xi_{T}$ be orthogonal to the trivials is equation (18a). For the time-independent $\xi_{T}$, we find from (3) that

$$
\Delta_{\xi} v_{x}=2 i \alpha \Omega(x+i y), \quad \Delta_{\xi} v_{y}=-2 \alpha \Omega(x+i y) ;
$$

and the orthogonality requirement becomes

$$
0=\nabla_{x} \Delta_{\xi} v_{y}-\nabla_{y} \Delta_{\xi} v_{x}=-2 \alpha \varpi \Omega^{\prime}(\varpi) e^{i m \phi} .
$$

In other words, as stated above, the displacement $\xi_{T}$ will be canonical only when the angular velocity $\Omega$ is constant.

\section{SECULAR INSTABILITY AGAINST VISCOSITY}

\section{a) Previous Criteria}

In their fundamental paper on stellar perturbation theory, Lynden-Bell and Ostriker (1967) remarked without proof that positivity of the operator $C^{i}{ }_{j}$ was a necessary and sufficient condition for secular stability against viscosity. In fact, their remark is ambiguous because it does not specify in which frame $C^{i}{ }_{j}$ is to be evaluated: their definition of $C^{i}$, includes the angular velocity of an arbitrary rotating frame. But it is clear from the later work of Tassoul and Ostriker (1968) that they had the inertial frame in mind. If this were correct, then the criterion for secular stability against viscosity would be identical to that for gravitational radiation. Unfortunately, as has been argued by Hunter (1977), this is not the case. The Lynden-Bell-Ostriker conjecture was based on the assumption that $E_{c}$ was the total energy associated with the perturbation, and must therefore decrease in time because of the dissipative action of the perturbation. In fact, however, we shall see in $\S$ IV below that $E_{c}$ actually increases for perturbations that are unstable to viscosity; the total energy may decrease with time, but that part represented by $E_{c}$ does not. On the other hand, if one defines the analogous energy $E_{c, R}$ in the rotating frame, one finds that this 
functional does decrease monotonically in time. Curiously, this fact seems to have been fully understood by early workers on secular instability, at least for systems with a finite number of degrees of freedom. It is perfectly clear, for example, in Lyttleton (1953, p. 23). It forms, as we shall see, the basis for the viscous stability criterion.

\section{b) The Rotating-Frame Criterion}

We confine our remarks to rigidly rotating stars with angular velocity $\Omega$; stability (to viscosity) of stars which are differentially rotating cannot be consistently defined in our approach since we deal only with unperturbed stars which are stationary and have purely axial velocity fields. Such stars cannot satisfy the viscous equations we use for the perturbations, so the perturbation theory would not be consistent. It is possible to have a differentially rotating locally stationary viscous solution if one permits small radial velocities as well: this is the case for accretion disks. There the global energy generation problem is taken care of by boundary conditions; such systems are outside our scope here.

If $E_{c}$ is the canonical energy defined in the inertial frame (as in $\S$ II), and if $J_{c}$ is the canonical angular momentum in the inertial frame, defined by

$$
J_{c}=-\int d V \rho \xi_{, \phi}^{i}\left(\xi_{i, t}+(v \cdot \nabla) \xi_{i}\right),
$$

then the canonical energy defined in the rotating frame (cf. Paper I) is

Moreover, remarkably, this is also

$$
E_{c, R}=E_{c}-\Omega J_{c} \text {. }
$$

$$
E_{c, R}=\delta^{2} E-\Omega \delta^{2} J,
$$

where $\delta^{2} E$ and $\delta^{2} J$ are the total second-order changes in the energy and angular momentum in the inertial frame. Equation (39) shows that $E_{c, R}$ is gauge-invariant, but it also shows something else. Since viscosity dissipates energy but conserves angular momentum, it causes the right-hand side of (39) to decrease in time. Therefore, as Hunter (1977) has shown, $E_{c, R}$-and not $E_{c}$-decreases monotonically under the action of viscosity. The argument is as follows.

The Navier-Stokes equation is

$$
\rho \partial_{t} v^{i}+\rho v \cdot \nabla v^{i}+\nabla^{i} p+\rho \nabla^{i} \Phi=2 \nabla_{j}\left(\eta \sigma^{i j}\right)+\nabla^{i}(\zeta \theta),
$$

where $\eta$ and $\zeta$ are the viscosity coefficients and where

and

$$
\theta=\nabla \cdot v
$$

$$
\sigma^{i j}=\frac{1}{2}\left(\nabla^{i} v^{j}+\nabla^{j} v^{i}\right)-\frac{1}{3} g^{i j} \theta .
$$

In the unperturbed star, the velocity field is shear and divergence free, and the right-hand side of equation (40) vanishes. Denoting by $F^{i}$ the right-hand side of equation (40) for the perturbed configuration, we have

$$
F^{i} \equiv \delta\left[2 \nabla_{j}\left(\eta \sigma^{i j}\right)+\nabla^{i}(\zeta \theta)\right]=2 \nabla_{j}\left(\eta \delta \sigma^{i j}\right)+\nabla^{i}(\zeta \delta \theta) .
$$

In the remainder of this section it will be helpful to change our notation slightly and use a dot to mean the time derivative in a rotating frame:

Then from (I.11) we obtain

$$
\dot{f}=\left(\partial_{t}+\Omega \partial_{\phi}\right) f .
$$

and thus

$$
\delta v^{i}=\partial_{t} \xi^{i}+v^{j} \nabla_{j} \xi^{i}-\xi^{j} \nabla_{j} v^{i}=\left(\partial_{t}+\Omega \partial_{\phi}\right) \xi^{i}=\dot{\xi}^{i}
$$

$$
F^{i}=2 \nabla_{j}\left(\eta \dot{\Sigma}^{i j}\right)+\nabla^{i}(\zeta \dot{\Theta}),
$$

where

and

$$
\Theta=\nabla \cdot \xi
$$

$$
\sum^{i j}=\frac{1}{2}\left(\nabla^{i} \xi^{j}+\nabla^{j} \xi^{i}\right)-\frac{1}{3} g^{i j \Theta} .
$$

The linearized perturbation equations are (see (I.15))

$$
F^{i}=A^{i} \partial_{t}{ }^{2} \xi^{j}+B^{i}{ }_{j} \partial_{t} \xi^{j}+C^{i}{ }_{j} \xi^{j}=A_{R}{ }_{j}^{i} \dot{\xi}^{j}+B_{R}{ }_{j}^{i} \dot{\xi}^{j}+C_{R}{ }^{i}{ }_{j} \xi^{j},
$$

where the operators $A_{R}, B_{R}$, and $C_{R}$ are defined by

and

$$
\begin{aligned}
& A_{R}=A, \\
& B_{R}=B-2 \Omega A \partial_{\phi},
\end{aligned}
$$

$$
C_{R}=C+\Omega^{2} A \partial_{\phi}{ }^{2}-\Omega B \partial_{\phi} .
$$


The canonical energy in a rotating frame was defined by equation (I.64) for solutions to equation (46) with $F^{i}=0$. Written in terms of the operators given above, it has the form

and its time derivative is

$$
E_{c, R}=\frac{1}{2}\left\langle\dot{\xi}, A_{R} \dot{\xi}\right\rangle+\frac{1}{2}\left\langle\xi, C_{R} \xi\right\rangle,
$$

$$
\frac{d}{d t} E_{c, R}=\langle\dot{\xi}, F\rangle
$$

Using equations (44) and (45) to express $F$ in terms of $\xi$, we find after integration by parts that

$$
\langle\dot{\xi}, F\rangle=\int \dot{\xi}_{i} F^{i} d V=\int-\left(2 \eta \dot{\Sigma}_{i j} \dot{\Sigma}^{i j}+\zeta \dot{\Theta}^{2}\right) d V,
$$

a negative semidefinite expression. Thus $E_{c, R}$ is a decreasing function of time and governs stability to viscosity in the sense that $E_{c}$ governs stability to radiation. That is:

$\mathrm{i}^{\prime)}$ If $E_{c, R}(\xi) \geq 0$ for all initial data, the configuration is stable in the sense that no perturbation dissipates infinite energy and the magnitude of $E_{c, R}$ is bounded.

ii") If $E_{c, R}(\xi)<0$ for some initial data $(\xi, \dot{\xi})$, then the configuration is unstable or marginally unstable in the presence of viscosity: there exist nonaxisymmetric perturbations which do not die away in time.

We expect that uniformly rotating stars will in fact be stable against viscosity if and only if $E_{c, R}$ is positive-definite.

\section{c) Local Secular Stability}

Rotating stars do not have a generic secular instability to viscosity at short wavelengths. The term in $E_{c}$ which caused the instability in the case of gravitational radiation, $-\rho|(v \cdot \nabla) \xi|^{2}$, is absent in $E_{c, R}$ (cf. eq. [51] below) and the condition for local stability is very simple. This corrects a misimpression that seems to have arisen from the work of Stewart (1975). He used the old $\left(E_{c}\right)$ criterion and found that the accretion disk was unstable to short wavelength modes, by an analysis similar to that given in § II above, but which took no account of the need to eliminate trivials. His result does not imply that there is any viscosity-induced instability, particularly nothing which would lead to turbulence. Since, as we have shown, $E_{c}$ remains of indefinite sign even after elimination of the trivials, one might be tempted to conclude that accretion disks at least have the gravitational radiation instability, but here we have two reservations. First, gravitational radiation should be weak compared to viscosity, and this should lead to a damping of the instability. (This might not be the case if disks had large coherent magnetic fields, since electromagnetic radiation excites the instability in the same way as gravitational radiation.) Second, if the accretion disk has a central rotating black hole, the criterion for instability given in $\S$ II above fails. Certain modes can extract energy from the hole and add it to $E_{c}$, so the sign of $d E_{c} / d t$ is not necessarily negative. This case needs to be examined separately. In contrast, the local criterion for viscous stability (of rigidly rotating fluids) is remarkably simple.

The Eulerian form of $E_{c, R}$ derived by Howard and Siegmann (1969) (cf. Paper I, Appendix C) in fact gives us a very convenient local stability criterion. The expression is

$$
E_{c, R}=\frac{1}{2} \int d V\left[\rho|\delta v|^{2}+\frac{1}{p \gamma}(\delta p)^{2}+\frac{1}{\rho}\left(\frac{\partial \rho}{\partial s}\right)_{p} \frac{d p}{d s}(\delta s)^{2}-\frac{1}{4 \pi G}|\nabla \delta \Phi|^{2}\right],
$$

where $\gamma$ is the adiabatic index and $d p / d s$ is the derivative of the relation $p=f(s)$ which obtains throughout the star by virtue of its rigid rotation. To study local stability, we set $\delta \Phi=0$. For normal fluids we have $\gamma>0$ and $(\partial \rho / \partial s)_{p}<0$. Then, since initial perturbations in $p$ and $s$ may be chosen independently, the stability of the star depends on the sign of $d p / d s:$ A rigidly rotating star is locally secularly stable against viscosity if and only if $s$ increases outward (i.e., in the direction of decreasing $p$ ). This is just the generalized Schwarzschild criterion.

\section{d) A Gauge Condition for Viscosity}

As we have said, the viscous stability criterion is gauge-invariant, so nothing more needs to be said if we are only interested in stability. It is interesting, however, that there exists a conserved gauge condition for viscous perturbations, one which will guarantee orthogonality of the perturbation to the trivials. This may be useful in studies of the detailed nature of the structure of unstable perturbations.

The gauge condition used in $\S$ II above was based on the symplectic inner product $W(\eta, \xi)$ introduced in Paper I (I.39):

$$
W(\eta, \xi)=\int\left[\eta_{i}\left(A_{j}^{i} \partial_{t} \xi^{j}+\frac{1}{2} B_{j}^{i} \xi^{j}\right)-\xi_{i}\left(A_{j}^{i} \partial_{t} \eta^{j}+\frac{1}{2} B_{j}^{i} \eta^{j}\right)\right] d V
$$


In order to adapt it to the viscous case, we first observe that $W$ has the same form in a rotating frame. That is, from equations (47) and (52) it follows that

$$
A^{i} \partial_{t} \xi^{j}+\frac{1}{2} B^{i}{ }_{j} \xi^{j}=A_{R}{ }_{j}^{i} \dot{\xi}^{j}+\frac{1}{2} B_{R}{ }^{i}{ }_{j} \xi^{j} .
$$

We shall rewrite $F^{i}$, defining a symmetric operator $F^{i}{ }_{j}$ by

$$
F^{i} \equiv F^{i}{ }_{j} \dot{\xi}^{j},
$$

with $F^{i}$ given in terms of $\xi^{i}$ by equations (44) and (45). It is then straightforward to show that if both $\xi$ and $\eta$ satisfy the dynamical equation (46), the time derivative of $W$ is given by

$$
\frac{d}{d t} W(\eta, \xi)=\int\left(\eta_{i} F_{j}^{i} \dot{\xi}^{j}-\xi_{i} F^{i}{ }_{j} \dot{\eta}^{j}\right) d V
$$

If $\eta$ is a trivial perturbation, then $\dot{\eta}^{i} \equiv 0$, and we have

$$
\frac{d}{d t}\left[W(\eta, \xi)-\int \eta_{i} F_{j}^{i} \xi^{j} d V\right]=0 .
$$

Thus, although there is apparently no conserved inner product between two general solutions of (46), there is one between an arbitrary solution $\xi$ and a trivial one $\eta$ :

$$
\tilde{W}(\eta, \xi)=W(\eta, \xi)-\int \eta_{i} F^{i}{ }_{j} \xi^{j} d V .
$$

(Notice that this, too, is frame-invariant, since the second term involves no time derivatives.) One can therefore use a conserved gauge condition: $\xi$ will be called a physical displacement if, for all trivial $\eta$,

$$
\tilde{W}(\eta, \xi)=0 \text {. }
$$

The complex version of this (for use with normal modes) is, since $F$ is symmetric, simply

$$
\tilde{W}(\eta, \xi)=W(\eta, \xi)-\int \eta_{i}{ }^{*} F^{i} \xi^{\xi} d V
$$

The condition this sets on $\xi$ is

$$
\epsilon^{i j k} \nabla_{i} s \nabla_{j}\left[\Delta_{\xi} v_{k}-\frac{1}{\rho} F_{k l} \xi^{l}\right]=0
$$

\section{SECULAR STABILITY OF NORMAL MODES}

\section{a) Basic Equations}

Because much analysis of stability is carried out by searching for normal modes, and since in many ways the results of $\S \S$ II and III above are more easily exhibited for normal modes, we feel it is worthwhile to devote some space to a discussion of the subject.

By a normal mode of oscillation of a nondissipative axisymmetric equilibrium, we mean a solution of the form

to the equation

$$
\begin{gathered}
\xi^{i}=\hat{\xi}^{i}(\varpi, z) e^{i(m \phi+\sigma t)} \\
A_{j}^{i} \partial_{t}{ }^{2} \xi^{j}+B^{i} \partial_{t} \xi^{j}+C^{i}{ }_{j} \xi^{j}=0 .
\end{gathered}
$$

We found in Paper I (eqs. [I.50]-[I.51]) that the conserved canonical energy and angular momentum of such a mode have the forms

and

$$
E_{c}=\sigma\left[(\operatorname{Re} \sigma)\langle\xi, A \xi\rangle-\frac{1}{2}\langle\xi, i B \xi\rangle\right]=\sigma[(\operatorname{Re} \sigma)\langle\xi, \rho \xi\rangle-\langle\xi, i \rho v \cdot \nabla \xi\rangle],
$$

$$
J_{c}=-m\left[(\operatorname{Re} \sigma)\langle\xi, A \xi\rangle-\frac{1}{2}\langle\xi, i B \xi\rangle\right]=-m[(\operatorname{Re} \sigma)\langle\xi, \rho \xi\rangle-\langle\xi, i \rho v \cdot \nabla \xi\rangle] .
$$

When the frequency $\sigma$ is real, we have

$$
E_{c} / J_{c}=\omega_{p},
$$

where $\omega_{p}=-\sigma / m$ is the pattern speed of the mode, the angular velocity of surfaces of constant phase. (When $\sigma$ is complex, $E_{\mathrm{c}}$ and $J_{c}$ vanish.) 
It will be useful for the discussion in $\S \mathrm{IV} b$ below to obtain an inequality satisfied by the canonical angular momentum $J_{c}$. From equation (61), when $\sigma$ is real,

Now

$$
\frac{J_{c}}{\langle\xi, \rho \xi\rangle}=-m\left[\sigma-\frac{\langle\xi, i \rho v \cdot \nabla \xi\rangle}{\langle\xi, \rho \xi\rangle}\right]
$$

and

From a Schwartz inequality

$$
v \cdot \nabla \xi^{i}=\Omega\left[i m \xi^{i}+\frac{1}{w} \xi^{w} \delta_{\phi}^{i}-\varpi \xi^{\phi} \delta_{w}^{i}\right]
$$

$$
-i \rho \xi_{i}^{*} v \cdot \nabla \xi^{i}=\rho \Omega\left[m|\xi|^{2}+i\left(\xi^{*} \times \xi\right)_{z}\right] \text {. }
$$

and we have

Thus, defining

$$
\left|\left(\xi^{*} \times \xi\right)_{z}\right| \leq|\xi|^{2},
$$

$$
\rho \Omega|\xi|^{2}(m-1) \leq-i \rho \xi_{i}^{*} v \cdot \nabla \xi^{i} \leq \rho \Omega|\xi|^{2}(m+1) .
$$

$$
\bar{\Omega}=\frac{\langle\xi, \rho \Omega \xi\rangle}{\langle\xi, \rho \xi\rangle},
$$

we obtain from equations (63) and (66) the inequality

$$
m^{2}\left(\omega_{p}-\bar{\Omega}-\frac{\bar{\Omega}}{m}\right) \leq \frac{J_{c}}{\langle\xi, \rho \xi\rangle} \leq m^{2}\left(\omega_{p}-\bar{\Omega}+\frac{\bar{\Omega}}{m}\right) .
$$

If $\sigma$ is complex, so that $E_{c}=J_{c}=0$, one finds in a similar way

$$
\bar{\Omega}\left(1+\frac{1}{m}\right) \geq \operatorname{Re}\left(\omega_{p}\right) \geq \bar{\Omega}\left(1-\frac{1}{m}\right) .
$$

When dissipation is present, the perturbation equations have the form

$$
A^{i} \partial_{t}{ }^{2} \xi^{j}+B_{j}^{i} \partial_{t} \xi^{j}+C^{i}{ }_{j} \xi^{j}=F^{i},
$$

where $F^{i}$ represents either the radiation reaction force or that due to viscosity. The canonical energy and angular momentum will no longer be conserved, their time dependence being given by the equations

$$
\frac{d}{d t} E_{c}=\operatorname{Re}\left\langle\partial_{t} \xi, F\right\rangle
$$

and

$$
\frac{d}{d t} J_{c}=-\operatorname{Re}\left\langle\partial_{\phi} \xi, F\right\rangle
$$

If there are solutions to (69) having harmonic time dependence, then equations (70) and (71) imply

$$
\frac{\left(d E_{c} / d t\right)}{\left(d J_{c} / d t\right)}=\omega_{p}
$$

where now the quantity $\omega_{p}$ may be complex. In other words, for normal modes in the dissipative case, $E_{c}$ and $J_{c}$ are locked together in a way analogous to that given by equation (62) for a conservative flow.

\section{b) Gravitational Radiation Instability of Normal Modes}

Because $d E_{c} / d t<0$ for any radiative solution to the perturbation equations including radiation reaction (or to the relativistic equations), no radiative oscillation which has harmonic time dependence and which is coupled to the radiation field can have purely real frequency. Instability in such a mode must therefore set in when its frequency vanishes (Friedman and Schutz 1975a), and one expects that any mode having $m \geq 2$ will in fact be radiative. The mode will be stable when $E_{c}>0$ and strictly unstable when $E_{c}<0$.

Now consider the onset of instability in some mode along a sequence of models that begins with a nonrotating star and along which some measure of the rotation increases. We assume that the mode and its characteristic frequency vary smoothly along the sequence and that in each model the angular velocity $\Omega$ is everywhere positive. The discussion will refer to a nondissipative Newtonian mode that approximates the exact radiative mode. For 
any $m>0$ the normal modes divide naturally in two classes, corotating $\left(\omega_{p}>0\right)$ and counterrotating $\left(\omega_{p}<0\right)$. When the rotation is small, equation (68) implies that (for modes whose frequencies remain finite as $\Omega \rightarrow 0$ ) corotating modes will have $J_{c}>0$ while counterrotating modes will have $J_{c}<0$. Thus by equation (62), both classes will have positive canonical energy and will be stable.

At the onset of instability in a mode, because its frequency vanishes, the inequality (68) implies that $J_{c}$ is negative -in fact, that $J_{c}<0$ in a neighborhood of the marginally stable model. Prior to this point of instability, $E_{c}$ is positive, and beyond the point $E_{c}$ is negative; therefore, again by equation (62), the pattern speed $\omega_{p}$ changes direction at the point of instability. The mode is counterrotating when stable and begins corotating when it becomes unstable. The unstable mode thus has $J_{c}<0$ but $\omega_{p}>0$.

This is the generic behavior of a large class of instabilities, because only two physical conditions are required to make it occur: coupling to some radiation field (which makes $\dot{E}_{c}<0$ ) and the existence of a background rotation which introduces a bias in the eigenfrequencies (counterrotating modes rotating slower than corotating ones). We call this class the rotational dragging instabilities.

We can now construct a rough picture of the generic instability in rotating stars. Since it exists for arbitrarily slow rotation, we can classify the modes as $p$ - and $g$-modes by analogy with the nonrotating star (cf. Ledoux and Walraven 1958, $\S 79$ ). The $p$-modes are acoustic modes, and for large $m$ (short wavelength) they are like sound waves. The $g$-modes are convective modes, dominated by entropy changes rather than density changes, and for short wavelength they approach zero frequency in a nonrotating star. They are absent (i.e., all zero-frequency and, in our terminology, part of the trivials) in an isentropic star. This explains why the speed of sound arises in the stability criterion of the isentropic star: the only modes are $p$-modes, and for high $m$ they travel at the speed of sound. They will go unstable if they are dragged supersonically by the rotation. The non-isentropic star, on the other hand, will have an instability not only in its $p$-modes but also in its $g$-modes. This is because the $g$-modes, approaching zero frequency for high $m$, are even easier to drag in the reverse direction than the $p$-modes. We can thus understand why the non-isentropic star is unstable for any trial function for sufficiently high $m$. However, we can probably safely conjecture that the $p$-modes of the nonisentropic stars are the important ones for instability, since they typically involve larger density changes than the $g$-modes do, and so will radiate gravitational waves more effectively.

\section{c) Viscosity-induced Instability of Normal Modes}

A brief discussion of normal modes in the presence of viscosity enables one to see more clearly why the canonical energy of the nonrotating frame is an inappropriate functional for viscous instability. We will make use of the angular momentum inequality (68) in which now $\bar{\Omega}=\Omega$ because we deal only with uniformly rotating stars.

For a normal mode, the viscous force $F^{i}$ of equation (44) has the form

and

$$
F^{i}=i(\sigma+m \Omega)\left[2 \nabla_{j}\left(\eta \Sigma^{i j}\right)+\nabla^{i}(\zeta \Sigma)\right]
$$

$$
\frac{d E_{c}}{d t}=-\operatorname{Re}\left\{\sigma^{*}(\sigma+m \Omega)\left[\left\langle\Sigma^{i j}, 2 \eta \Sigma_{\mathrm{ij}}\right\rangle+\langle\Sigma, \zeta \Sigma\rangle\right]\right\}
$$

For real frequency modes (modes stable in the absence of viscosity), we therefore find that

$$
\operatorname{sign}\left(\frac{d E_{c}}{d t}\right)=\operatorname{sign}\left[\omega_{p}\left(\Omega-\omega_{p}\right)\right],
$$

and thus $d E_{c} / d t$ has no definite sign.

We now construct an argument like the previous one for gravitational radiation. For small $\Omega$ (and for $\omega_{p}$ not limiting to 0 ), both counterrotating and corotating modes have $E_{c}>0$ and $d E_{c} / d t<0$, so both are stable. The mode that went unstable to gravitational radiation remains stable to viscosity, because although $E_{c}$ changes sign, so does $\omega_{p}$ and hence by equation (73), $d E_{c} / d t$ changes sign as well. In other words, the magnitude of $E_{c}$ remains a decreasing function of time. On the other hand, it may happen that (as in the Maclaurin sequence) $\Omega$ increases along the sequence faster than the pattern speed of some corotating mode. When they become equal, the sign of $d E_{c} / d t$ changes (while that of $E_{c}$ remains the same) and the mode goes unstable. Thus for the unstable mode, viscosity has the effect of making an already positive canonical energy $E_{c}$ increase in time. Similarly, although viscosity preserves the real angular momentum of the star, it causes the canonical angular momentum $J_{c}$ to increase for an unstable star. ${ }^{5}$

It is evident from this discussion that the sign of $E_{c}$ is irrelevant to the question of viscosity-induced instability.

${ }^{5}$ This may seem puzzling in view of the fact that one can represent a perturbation at any time by canonical data for which $J_{c}$ is equal to the second order change in the system's angular momentum. As was pointed out in $\S$ IV $b$ above, however, the time evolution in the presence of viscosity of canonical initial data will not be a canonical displacement; thus $J_{c}$ will not remain the physical angular momentum of the perturbation. 


\section{d) Orthogonality Properties of Normal Modes}

We will show that smooth normal modes of the nondissipation system are in general canonical: orthogonal to the trivials with respect to the inner product $W$. The only possible exceptions are in cases where $\nabla \Omega$ vanishes at corotation points of the mode. In addition, we will see that normal modes with different frequencies are orthogonal to each other in $W$.

The second statement is the simplest. If

$$
\xi_{1}=\hat{\xi}_{1} \exp \left[i\left(m \phi+\sigma_{1} t\right)\right] \quad \text { and } \quad \xi_{2}=\hat{\xi}_{2} \exp \left[i\left(m \phi+\sigma_{2} t\right)\right]
$$

are normal modes, their inner product with respect to $W$ is given by (see [1] or [I.39])

$$
\begin{aligned}
W\left(\xi_{1}, \xi_{2}\right) & =\int \rho\left[\xi_{1}{ }^{*}\left(\partial_{t} \xi_{2 j}+v \cdot \nabla \xi_{2 j}\right)-\xi_{2}{ }^{j}\left(\partial_{t} \xi_{1 j}{ }^{*}+v \cdot \nabla \xi_{1 j}{ }^{*}\right)\right] d V \\
& =\int \rho\left[\hat{\xi}_{1}{ }^{*}\left(i \sigma_{2}+i m \Omega+v \cdot \nabla\right) \hat{\xi}_{2 j}-\hat{\xi}_{2}{ }^{j}\left(-i \sigma_{1}{ }^{*}-i m \Omega+v \cdot \nabla\right) \hat{\xi}_{1 j}{ }^{*}\right] \exp \left[i\left(\sigma_{2}-\sigma_{1}{ }^{*}\right) t\right] d V .
\end{aligned}
$$

But $W$ is independent of time, whence $W=0$ unless $\sigma_{1}^{*}=\sigma_{2}$.

In a similar way, we show that a twice-differentiable normal mode $\xi=\hat{\xi}(\varpi, z) e^{i(m \phi+\sigma t)}$ is orthogonal to all trivials except if $\sigma$ is real and the mode has a corotation point $\left(\omega_{p}=\Omega\right)$ where $|\nabla \Omega|=0$. Any trivial $\eta$ can be written as (cf. [I.22])

$$
\rho \eta^{j}=\epsilon^{j k l} \nabla_{k} s \nabla_{l} f,
$$

where $f=f(\varpi, z, \phi-\Omega t)$. For $f$ to have the correct angular dependence it must be expressible as

$$
f=\hat{f}(\boldsymbol{w}, z) e^{i m(\phi-\Omega t)},
$$

where of course $\Omega$ may be a function of $\varpi$ and $z$. For a trivial, two integrations by parts of equation (1) give (I.67):

$$
\begin{aligned}
W(\xi, \eta) & =-\int \hat{f}(\varpi, z) e^{i m(\phi-\Omega t)} \epsilon^{i j k} \nabla_{j} s \nabla_{k}\left\{\left[i\left(\sigma^{*}+m \Omega\right) \hat{\xi}_{i}^{*}+i m \Omega \delta_{i}^{\phi} \hat{\xi}_{\phi}{ }^{*}-v^{l}\left(\nabla_{l} \hat{\xi}_{i}{ }^{*}+\nabla_{i} \hat{\xi}_{l}\right)\right] e^{-i m \phi}\right\} e^{-i \sigma^{*} t} d V, \\
& =\int g(\varpi, z) e^{-i\left(\sigma^{*}+m \Omega\right) t} d V .
\end{aligned}
$$

This equation defines $g(\varpi, z)$. Since $W$ is again time-independent, it must vanish if $\sigma$ is complex. If $\sigma$ is real, then we integrate:

$$
\int_{-T}^{T} W d t=2 W T
$$

an expression which diverges as $T \rightarrow \infty$ unless $W=0$. On the other hand,

$$
\int_{-T}^{T} \int g(\varpi, z) e^{-i(\sigma+m \Omega) t} d V d t=\int g(\varpi, z) \int_{-T}^{T} e^{-i(\sigma+m \Omega) t} d t d V
$$

and for large times

$$
\int_{-T}^{T} e^{-i(\sigma+m \Omega) t} d t \approx 2 \pi \delta(\sigma+m \Omega) .
$$

The argument of the $\delta$ function vanishes except on surfaces of corotation, $S_{\alpha}$, satisfying $\Omega(w, z)=\omega_{p}$. We have

$$
\begin{aligned}
\int g(\varpi, z) \delta(m \Omega+\sigma) d V & =\sum_{\alpha} \int_{S_{\alpha}} \frac{g}{\left|m n_{\alpha} \cdot \nabla \Omega\right|} d S \\
& =\frac{1}{|m|} \sum_{\alpha} \int_{S_{\alpha}} \frac{g}{|\nabla \Omega|} d S,
\end{aligned}
$$

where $n_{\alpha}$ is the unit normal to the surface $S_{\alpha}$. Thus the limiting value of the integral in equation (77) is well defined if at corotation points $\nabla \Omega \neq 0$.

The fact that normal modes are usually canonical should not be surprising. Trivials are generated by functions of $\phi-\Omega(\varpi, z) t$, so they have a continuous spectrum of pattern speeds spanning the range of $\Omega$ in the star. A mode with a pattern speed outside this range - that is, having no corotation point - can obviously contain no 
trivial part. If $|\nabla \Omega| \neq \dot{0}$ everywhere (the usual case for differential rotation), then no trivial defined on an open set in $\varpi$ and $z$ can have a single pattern speed and so cannot be part of even a normal mode whose pattern speed allows a corotation point. The exception here is a normal mode which is not twice-differentiable. This may be important: the work of Hunter (1969) and Bardeen (1975) on perturbations of rotating disks shows that, at least under certain conditions, a real-frequency eigenfunction $\hat{\xi}(\varpi, z)$ will have discontinuous first derivatives at a corotation point. (Such discontinuities are well known in hydrodynamics; see, for example, Rayleigh 1945 and Chandrasekhar 1961.) This means that $g(\varpi, z)$ in $(76)$ may contain $\delta$-functions at corotation, picking out a finite piece of $W(\xi, \eta)$ which is explicitly time-independent, and showing that the eigenfunction might contain a trivial piece. It should still be possible, however, to add a trivial also having discontinuous first derivatives at corotation to the eigenfunction to produce one for which $W(\xi, \eta)$ vanished for all $\eta$. This would then be a purely canonical mode.

Similar remarks apply to the rigidly rotating star, where $|\nabla \Omega|=0$ everywhere. In this case all trivials have pattern speed $\Omega$, so a mode with the same pattern speed can contain an arbitrary trivial part. Again, the results of Paper I encourage us to believe that it will usually be possible to find a canonical mode with the same eigenfrequency.

In this way one expects to be able to construct a full set of canonical eigenmodes with discrete frequencies. One would hope that this set plus the trivials would usually form a complete set of eigenmodes for the solutions of the dynamical equation, (I.15). The remarkable thing, which seems not to have been appreciated before, is that most of the eigenmodes will automatically satisfy the additional equation.

$$
\epsilon^{i j k} \nabla_{i} s \nabla_{j} \Delta_{\xi} v_{k}=0
$$

\section{QUESTIONS FOR THE FUTURE}

Because the work reported here substantially revises previous notions of secular stability, it suggests a number of new questions that ought to be answered. We will list some of these here.

1. When the quantity $\nabla \alpha \times \nabla s=0$ somewhere in a star, the canonical displacements apparently do not describe all physical perturbations. How does one phrase a sufficient condition for stability in this case? A related problem is that the definition of a canonical displacement is vacuous if $\nabla \alpha \times \nabla s$ vanishes in some three-dimensional region of a star. Can this occur, and, if it can, is there some further condition that can be imposed?

2. It is unclear from the form of the canonical energy $E_{c}$ that its minimum value for (normalized) canonical initial data $(\dot{\xi}, \xi)$ can be found by restricting oneself to time-independent trial functions (data with $\dot{\xi}=0)$. On the other hand, if one assumes that instability sets in via a discrete normal mode, the data marking the point of marginal stability will be time independent because those data give rise to a zero-frequency mode. Is there an argument independent of normal mode assumptions that would allow one to dispense with time-dependent trial functions?

3. What is the time scale of the generic gravitational radiation instability in isentropic stars? (The estimate given in $\S$ II above is probably rougher than order of magnitude.)

4. There is generally a unique correspondence between canonical displacements and physical perturbations:

$$
(\dot{\xi}, \xi) \leftrightarrow\left(\delta p, \delta \rho, \delta v^{i}, \delta s\right)
$$

It is therefore in principle possible to express the functional $E_{c}$ that governs secular stability to gravitational radiation in terms of Eulerian perturbations. What is the expression, and is there a natural way to derive it that uses only the Eulerian changes in the fluid variables?

5. In the Maclaurin spheroids, the secular instabilities to viscosity and to radiation reaction for $m=2$ set in at the same point. It seems clear that this is a coincidence: is it false for the analogous $m=2$ modes of uniformly rotating compressible models and does it hold true for modes with $m>2$ in Maclaurin?

6. The key term in $E_{c}$ responsible for the generic instability of rotating stars to radiation is $\int\left(-p|v \cdot \nabla \xi|^{2}\right) d V$. That term is not present in the functional $E_{c, R}$ which governs stability against viscosity, and one consequently expects that dynamically stable models in slow uniform rotation will be stable in the presence of viscosity. Is this true?

7. We have presented arguments that our conditions for secular instability are sufficient for strict instabilitythat they imply more than the existence of a marginally stable deformation. Can one construct a rigorous proof, perhaps along the lines of Laval, Mercier, and Pellat (1965)?

8. The coupling to electromagnetic radiation that results from strong magnetic fields is commonly more important than the coupling to gravitational radiation, and it should induce the same kind of instabilities, including the generic instability. In what stars are they important?

9. We have excluded differentially rotating stars from our discussion of viscosity, but it might be possible to phrase a consistent secular stability criterion under two circumstances: if the unperturbed star were locally stationary because it had radial flow, and if viscosity would drive instabilities on a faster time scale than it would cause redistribution of angular momentum in the unperturbed star. What are the relevant criteria?

10. We have treated viscosity and gravitational radiation separately, but in the real world they are usually both present. Moreover, they tend to stabilize each other (Lindblom and Detweiler 1977). Is it possible to find a single 
criterion for secular instability which includes both effects? We are not convinced this is possible; one may always simply have to use a normal-mode calculation.

We gladly acknowledge helpful conversations and correspondence with J. Bardeen, S. Chandrasekhar, L. Howard, R. Sorkin, and S. Teukolsky. B. F. S. thanks the Royal Society of London and University College, Cardiff for travel grants, and Cornell University for its hospitality while this work was in progress.

\section{REFERENCES}

Bardeen, J. M. 1975, in IAU Symposium No. 69, Dynamics of Stellar Systems, ed. A. Hayli (Dordrecht: Reidel).

Bardeen, J. M., Friedman, J. L., Schutz, B. F., and Sorkin, R. 1977, Ap. J. (Letters), 217, L49.

Chandrasekhar, S. 1961, Hydrodynamic and Hydromagnetic Stability (Oxford: Clarendon Press), § 103.

- 1970, Phys. Rev. Letters, 24, 611.

Comins, N., and Schutz, B. F. 1978, submitted to Proc. $R$. Soc. $A$.

Durisen, R. H. 1975, Ap. J., 199, 179.

Friedman, J. L. 1975, unpublished.

Friedman, J. L., and Schutz, B. F. 1975a, Ap. J., 200, 204. . 1975b, Ap. J. (Letters), 199, L157. 1978, Ap. J., 222, in press (Paper I)

Howard, L. N., and Siegman, W. L. 1969, Studies Appl. Math., 48, 153.

Hunter, C. 1969, Studies Appl. Math., 48, 55. 1977, Ap. J., 213, 497.

Laval, G., Mercier, C., and Pellat, R. 1965, Nucl. Fusion, 5, 156.

John L. Friedman: Physics Department, University of Wisconsin-Milwaukee, Milwaukee, WI 53201

Bernard F. Schutz: Department of Applied Mathematics and Astronomy, University College, P.O. Box 78, Cardiff CF1 1XL, Great Britain
Ledoux, P., and Walraven, Th. 1958, Handbuch der Physik (Berlin: Springer-Verlag), Vol. 51, pp. 353-604.

Lindblom, L., and Detweiler, S. L. 1977, Ap. J., 211, 565.

Lynden-Bell, D., and Ostriker, J. P. 1967, M.N.R.A.S., 136, 293.

Lyttelton, R. A. 1953, The Stability of Rotating Liquid Masses (Cambridge: Cambridge University Press)

Ostriker, J. P., and Bodenheimer, P. 1973, Ap. J., 180, 159.

Ostriker, J. P., and Peebles, P. J. E. 1973, Ap. J., 186, 467.

Ostriker, J. P., and Tassoul, J.-L. 1969, Ap. J., 155, 987.

Rayleigh, Lord, 1945, Theory of Sound (New York: Dover), Vol. 2, chap. 21.

Roberts, P. H., and Stewartson, K. 1963, Ap. J., 137, 777.

Stewart, J. M. 1975, Astr. Ap., 42, 95-101.

Tassoul, J.-L. and Ostriker, J. P. 1968, Ap. J., 154, 613.

Thompson, W., and Tait, P. G. 1883, Principles of Natural Philosophy, Vol. 2 (Oxford: Clarendon Press). 